Appeared in Journal of Circuits, Systems and Computers, vol. 6, no. 2, pp. 115-139, April 96 (C)

\title{
Implementation and Experimental Results of a Chip for The Separation of Mixed and Filtered Signals *
}

\author{
Ammar B. A. Gharbi and Fathi M. A. Salam \\ Circuits, Systems and Artificial Neural Networks Laboratory \\ Department of Electrical Engineering \\ Michigan State University, East Lansing, MI 48824 \\ email: gharbi@ee.msu.edu, salam@ee.msu.edu
}

\begin{abstract}
We describe an algorithm and chip implementation for separating a mixture of unknown, but independent, temporal signals in static and dynamic environments. The proposed algorithm, which is a simple modification of the Herault and Jutten (HJ) algorithm, proved to be robust to parameter variations in both cases. We present chip test results to quantify the performance of the modified algorithm in static and dynamic (mixing and filtering) environments. Moreover, experiments of separating two speech or two music signals in English-English, English-Japanese, and English-Arabic have demonstrated successful performance.
\end{abstract}

*Acknowledgment: This work is supported in part by the Michigan Research Excellence Fund (REF). 


\section{Introduction}

The Separation of Independent Sources (SIS) is a challenging problem with significant potential applications. The problem is informally described as follows: several unknown but independent temporal signals propagate through a mixing and/or filtering, natural or synthetic, medium. By sensing outputs of this medium, a network (e.g., a neural network, a system, or a device) is configured to counteract the effect of the medium, and adaptively recovers the original, unmixed, signals. Only the property of signal independence is assumed for this processing. No additional apriori knowledge of the original signals is required. This processing represents a form of self (or unsupervised) learning. The weak assumptions and self-learning capability render such a network attractive from the viewpoint of real-world applications. See $[1,2,3]$ for further descriptions.

This neural network approach to the signal processing problem of SIS has great advantages over the existing adaptive filtering algorithms. For example when the mixture of other signals are labeled as noise in this approach, no specific apriori knowledge about any of the signals is assumed; only that the processed signals are independent. This is in contrast to the noise cancelation method, proposed by Widrow et al. [4], which requires that a reference signal be correlated exclusively to the part of the waveform (i.e. noise) that needs to be filtered out. This latter requirement entails specific apriori knowledge about the noise as well as the signal(s).

The separation of independent sources is valuable in numerous and major applications in areas as diverse as telecommunication systems, sonar and radar systems, audio and acoustics, image/information processing, and biomedical engineering. Consider, e.g., the audio and sonar scenario when the original signals are sounds and the mixed signals are the output of several microphones or sensors placed at different vantage points. A network will receive, via each microphone, a mixture of sounds that are usually delayed relative to one another and/or the original sounds. The network's role is then to dynamically reproduce the original signals, where each separated signal can be subsequently channeled for further processing or transmission. Similar application scenarios can be described in situations involving heart-rate measurements, communication in noisy environments, engine diagnostics, and uncorrupted cellular phone communications.

When the sensors are viewed as part of the medium, adequate models of the medium that include time delays or filtering are very important. In many applications where the sensors (e.g., microphones) include their own dynamics, the model of medium ought to include time-delays or filtering. Otherwise, assuming pure static models of the medium, would result in highly sensitive 
and unrobust processing for any network. Indeed, in order to render the network operable in realworld applications, robust operations must be ensured to parameter variations, dynamic influences and signal delays that often result in asynchronous signal propagation. Examples of such effects include delay lines and/or echoes in the case of a radar system, and filters in the case of an audio system. The medium should be indeed modeled as a dynamic linear (or even, nonlinear) system $[5,6]$.

In $[1,2]$, a neuro-inspired algorithm is proposed for the SIS problem. The algorithm is also explained from the viewpoint of statistical signal processing but is largely heuristic. For instance, global stability analyses are lacking. Yet, the algorithm's performance has been demonstrated by successful prototype simulations. The algorithm, however, does not consider dynamic medium or delays. It considers a static mixing of signals, where each output of the medium entails one signal and a fraction of each other signal. The simulation evidence has focused primarily on consideration of 2-signal prototypes. Examples have been reported in $[7,8]$ as well. However, the algorithm fails to perform separation of sources in case the medium is no longer a static linear model. Also, it lacks robustness to parameter variations and to the presence of dynamics or filtering in the medium.

Thus far, the separation of independent sources has been implemented in CMOS technology using static linear models of the medium. Successful testing of these implementations have been reported in $[7,8]$. However, these implementations cannot perform separation of sources in realistic environments (which include dynamics) due to the limited nature of the algorithm.

This paper describes a modification of the Herault-Jutten (HJ) algorithm which aspires to address the effect of dynamic media in filtering and delaying signals. Thus the network model includes dynamics in order to accommodate the ever-present dynamics in the mixing medium. The capability of the modification is verified in static and dynamic media by numerous computer simulation results. Its performance is found to be superior to the original HJ algorithm. The paper also describes the robust CMOS implementation of the modification. Our implementation is similar to the one proposed in [7]. However, time-constants and time-scales are carefully considered to satisfy time-scale separation criteria. The paper provides extensive performance studies on several applications of a prototype neuro-chip. These studies include static and dynamic media where we experimentally quantify the chip's capability and limitations. Thorough testing of the chip is performed using prototype signals as well as real-time, real-world, signals such as speech or music waveforms that include English, Japanese, and/or Arabic.

The paper is organized as follows. Section 2 defines the problem of separation of indepen- 
dent sources (SIS) and summarizes the HJ algorithm. Section 3 describes the modification of the algorithm and its motivation. Section 4 discusses and compares computer simulations of both the algorithm and its modification, demonstrating the advantages of the latter. Section 5 presents the basic building blocks used to implement the proposed algorithm. Section 6 summarizes the experimental results of a fabricated CMOS chip that include several speech and music separation experiments. Finally, some concluding remarks are given in section 7 .

\section{Problem Definition}

The general block diagram for SIS is shown in Figure 1. The vectors $s(t), e(t)$, and $y(t)$ are, respectively, the unknown source vector, the measured signal vector, and the output signal vector. The neural network to be designed receives the signal $e(t)$ as its input and adaptively modifies $y(t)$ to reproduce the original signal $s(t)$.

In $[1,2,9]$, Herault and Jutten had proposed an algorithm for the separation of independent sources. It is assumed that the medium is static and linear. The inputs to the network are the measured signals $e_{i}(t), 1 \leq i \leq n$, which are linear combinations of the original signals, namely,

$$
e_{i}(t)=\sum_{i=1}^{n} a_{i j} s_{j}(t)
$$

In vector form, one has

$$
e(t)=A s(t)
$$

$A$ is an $n \times n$ matrix whose components $a_{i j}$ are unknown. $A$ models the the mixing static environment and is assumed to be nonsingular. Furthermore, for normalized mixing, its diagonal entries are all ones and each off- diagonal element is less than one in absolute value. Herault and Jutten used a recursive architecture made up of fully interconnected outputs. Each output, $y_{i}(t), 1 \leq i \leq n$, receives the mixed signal, $e_{i}(t)$, and a weighted sum of all other outputs, $-\sum_{j \neq i} d_{i j} y_{j}(t)$. Thus,

$$
y_{i}(t)=e_{i}(t)-\sum_{j \neq i} d_{i j} y_{j}(t) \quad 1 \leq i \leq n
$$

which, in vector form, becomes the (static) network:

$$
y(t)=e(t)-D y(t)
$$

$\mathrm{D}$ is an $n \times n$ weight matrix whose main diagonal is zero. Now, the problem of separation of signals translates to retrieving the original signals $s(t)$. In the limit, it is thus desired to have:

$$
y(t)=P s(t)
$$


where $\mathrm{P}$ is a permutation matrix ( i.e. a matrix obtained from the identity by row and/or column permutation).

Herault and Jutten were motivated by some neurobiological evidence that the information about the speed and position of body joints are mixed before being sent to the brain by two different types of nerves. The brain is however perfectly capable of separating (and recovering) speed and position signals. This biological and intuitive inspiration led Herault and Jutten to propose an update law that presumes the independence of the original signals:

$$
\dot{d}_{i j}=\eta_{i j} f\left(y_{i}\right) g\left(y_{j}\right) \quad i \neq j
$$

where $f($.$) and g($.$) are two nonlinear odd functions and \eta_{i j}$ is a constant learning rate. (We differ details of the update law (3) to their original work in $[1,2]$.) This algorithm has been implemented in CMOS. Successful testing of several implementations, using static models of the mixing medium and the network described by (1) and (2), have been reported in [7] and [8]. However, the algorithm, and thus its implementations, lacks robustness to changes in the model of the medium (which, in practice, includes dynamics and time-delays). Next, we propose a modification of the algorithm that embraces dynamics in the medium and is robust to parameter and filtering variations.

\section{A Modified HJ Algorithm}

The present HJ network governed by the set of equations (1-3) assumes a static environment model as well as a static network. The network model, described by (2), can be rewritten as

$$
0=-y_{i}+e_{i}-\sum_{j \neq i} d_{i j} y_{j}
$$

To include the effect of the transient dynamics, we consider the dynamic network model

$$
\tau_{i} \dot{y}_{i}(t)=-y_{i}(t)+e_{i}(t)-\sum_{j \neq i} d_{i j} y_{j}(t)
$$

where $\tau_{i}$, for all $i$, is selected to to be small enough to provide time-scale separation between (3) and (4). Observe that equation (4) is a differential equation in the output variable with sufficiently fast dynamics. Thus the augmented state space of (3) and (4) includes the output variables $y_{i}(t)$ as states. In this contest, the augmented dynamical system will qualitatively retain only the limit sets which are robust in the augmented state space of $y_{i}(t)$ and $d_{i j}(t)$. This robustness property is important in view of the fact that physical circuit realization is necessarily prone to function 
approximation and inaccuracies. Moreover, this modified algorithm performs smoothing and enables an integrated circuit implementation to dominate the ever-present parasitic capacitances of its transistors. The modified algorithm [5] was shown to be more robust than the original HJ algorithm to parameter and filtering variations. Simulation results, shown below, will demonstrate that the system described by (1), (3) and (4) successfully separates mixed signals more robustly. Our chip implementation of system (3) and (4), discussed below, will be tested in both the static medium case as well as the more realistic dynamic medium case.

\section{Computer Simulations}

Computer simulations were performed to study the performance of the proposed algorithm compared to the original algorithm. The two nonlinear functions $f($.$) and g($.$) are chosen to be$

$$
f(x)=\sinh x \quad \text { and } \quad g(x)=\tanh x
$$

The proposed algorithm will converge to the desired parameters, $a_{i j}$, and retrieves the original signals if and only if time-scale separation between the equations (4) and (3) is maintained. Thus, a necessary condition between the parameter learning rates, $\eta_{i j}$, and the time constants, $\tau_{k}$, must be satisfied, namely,

$$
\eta_{i j} \tau_{k} \ll 1 \quad \forall i, j, k
$$

This condition will also guarantee that the rate of change of the dynamical state $y(t)$ is faster than that of the update of the parameters $d_{i j}$.

Figure 2 shows the simulations for a 2 -dimensional network with $\eta=0.05$ and $\tau=0.001$ (satisfying the above condition). The original signals, which are two sine functions with different frequencies, were mixed as described in (1) for different static mixing combinations including $a_{12}=$ 0.7 and $a_{21}=0.3$. Both algorithms converge to the desired parameters $a_{i j}$. However, the output $y(t)$ of the network model is still a mixture of the original signal for the original HJ algorithm whereas the output $y(t)$ of the modified algorithm is a replica of the original signal.

We have conducted numerous computer simulations for $2,3,4$, and 5 dimensional networks. All computer simulations give a validation of the robust performance of the proposed modification. We next describe the micro-electronic implementation of the modified algorithm. 


\section{CMOS Micro-electronic Implementation}

The modified HJ algorithm is implemented in CMOS using $2.0 \mu m$ technology on a Tiny Chip $(2.22 \times$ $2.25 \mathrm{~mm}^{2}$ ). Similar building blocks to that of the Vittoz-Arreguit [7] implementation are used with the modification in the summer circuit to realize the implementation of (4). The basic components of the circuit are a transconductance amplifier [10] and a sine hyperbolic circuit [7]. In Figure 3, the circuit diagrams of the basic components and their corresponding measured characteristics in the subthreshold regime are shown. These basic components produce a relationship between the input voltage $\mathrm{V}$ and the output current, which is expressed in units of $\frac{k T}{q}$ (see $[10,7]$ ):

$$
\begin{aligned}
I_{\text {out }}^{\text {tanh }} & =I_{b} \tanh \frac{\kappa}{2} V \\
I_{\text {out }}^{\text {sinh }} & =2 w I_{0} e^{\kappa\left(V_{b}+\delta V\right)} \sinh \kappa V
\end{aligned}
$$

The voltage $V$ is referenced to the ground level (i.e. $V_{\text {ref }}=0.0$ Volts). The rail voltages are at \pm 2.5 Volts. It is important to observe that some of the parameters that the above currents depend on are: the biasing voltage $V_{b}$; the ratio of width of the biasing transistor to the width of the arm transistors $w$; and the offset voltage $\delta V$. Equations (3) is implemented in CMOS by the circuit diagram shown in Figure 4(a):

$$
\dot{V}_{i j}=\frac{2 w I_{0} e^{\kappa\left(V_{b}+\delta V\right)}}{C_{i j}} \sinh \kappa V_{i} \tanh \frac{\kappa}{2} V_{j}
$$

Using Kirchoff's current law at the inverting node of the amplifier shown in Figure 4(b):

$$
\bar{C}_{i} \dot{y}_{i}+I_{d s T_{1}}+I_{d s T_{2}}+\sum_{j \neq i} I_{d s T_{i j}}=0
$$

In the linear region, the drain-to-source current depends on the drain-to-source voltage $v_{d s}$ and the gate-to-source voltage $v_{g s}$

$$
I_{d s}\left(v_{d}, v_{s}, v_{g}, V_{T}\right)=\beta\left[\left(v_{g s}-V_{T}\right) v_{d s}-\frac{v_{d s}^{2}}{2}\right]
$$

where $\beta$ and $V_{T}$ are respectively the transconductance parameter and the threshold voltage of the transistor. This circuit will operate in the linear region if the gate voltage of the transistors $T_{1}$ and $T_{2}$ is much larger than the peak value of the input $e_{i}$. By replacing the expression of the drain-to-source current in the later equation, we obtain

$$
\bar{C}_{i} \dot{y}_{i}=\beta\left(V_{R}-V_{T}\right)\left(e_{i}-y_{i}\right)-\beta \sum_{j \neq i}\left(V_{i j}-V_{T}\right) y_{i}+\beta \frac{e_{i}^{2}+\sum_{j} y_{i}^{2}}{2}
$$


Thus equation (4) is implemented in CMOS approximately as

$$
\frac{\bar{C}_{i}}{\beta\left(V_{R}-V_{T}\right)} \dot{y}_{i}=e_{i}-y_{i}-\sum_{j \neq i} \frac{V_{i j}-V_{T}}{V_{R}-V_{T}} y_{i}+\frac{e_{i}^{2}+\sum_{j} y_{i}^{2}}{2\left(V_{R}-V_{T}\right)}
$$

In our implementation of (4), the square terms of $e_{i}$ and $y_{i}$ are present. But they can be neglected since the signals $e_{i}(t)$, and consequently $y_{i}(t)$, are small. The CMOS expression for the parameter $d_{i j}$ is

$$
d_{i j}=\frac{V_{i j}-V_{T}}{V_{R}-V_{T}}
$$

The table below shows the parameters in equations (3) and (4), their corresponding CMOS expressions as well as their nominal values in the course of testing.

\begin{tabular}{||l|c|c||}
\hline & CMOS expression & Nominal value \\
\hline$\tau_{i}$ & $\frac{\bar{C}_{i}}{\beta\left(V_{R}-V_{T}\right)}$ & $1.2 \times 10^{-8} s$ \\
$\eta_{i j}$ & $\frac{2 w I_{0} e^{\kappa\left(V_{b}+\delta V\right)}}{C_{i j}\left(V_{R}-V_{T}\right)}$ & $8.0 \times 10^{4} s^{-1}$ \\
\hline
\end{tabular}

Using these basic building blocks, the circuit implementation of the modified algorithm, governed by (3) and (4), is now realized. Observe that the ratio between $\tau_{i}$ and the inverse of $\eta_{i j}$ is about 4-folds, leading to a fast and slow time-scales of the dynamics of the network and the weight update. Circuit simulations of our CMOS implementation of the proposed algorithm using PSPICE were performed prior to the layout and fabrication stages. A sample of the obtained results of separating two sinusoidal signals of different frequencies is shown in Figure 5.

\section{Test Results}

The resulting chip is tested to quantify its performance for static and dynamic media. The tests are carried out for prototype signals as well as for real-world, real-time signals such as speech, music, and noise. The tests will be focused on studying the separation of signals and the robustness of the performance to variations in filtering and parameters.

\subsection{The Static Medium Case}

It is assumed here that the mixed signals are linear combinations of the unknown sources as described in (1). The chip testing is carried out using a two-neuron network where it is desired to separate two independent signals. 


\subsubsection{The Experimental Setup}

The combined circuits shown in Figure 6 , with $C_{F i j}=0$, produces a linear combination of the original sources. Summing all the currents at node 1 in Figure 6(b) gives

$$
e_{i}=\sum_{j=1}^{n} \frac{R_{0}}{R_{i j}} s_{j}=s_{i}+\sum_{j \neq i} \frac{R_{0}}{R_{i j}} s_{j}
$$

since $R_{i i}=R_{0}=10 k \Omega$. Thus, the coefficient of the matrix A in (1), $a_{i j}=\frac{R_{0}}{R_{i j}}$, can be varied by using an external variable potentiometer for $R_{i j}$. Such variations can be used to study the robustness of the network realized on the Tiny Chip.

\subsubsection{Experimental Results}

Experiments were conducted to investigate the performance of the chip for the separation of the signals in five scenarios: (i) separation of prototype functions (sine, square and triangle), (ii) separation of two segments of music signals (Japanese and English) from two different sources, (iii) separation of two speech segments of two different English speakers, (iv) separation of two speech segments of two different Arabic and English speakers, and (v) separation of white noise and an English speech segment. Successful separation was obtained in the first four cases for mixing levels less than $80 \%$. For the fifth case, separation was attained only for mixing levels less than $30 \%$. Above these mixing levels, the outputs of the network are not separated satisfactorily but rather contain a mixture of the original signals. Sample chip test results are shown for each of the five scenarios in Figures 7-10.

For the five mentioned scenarios, the level of mixing has been varied in order to study the robustness of the network to variations. For this reason, the parameters $a_{i j}$ are initially fixed to some values. Once the network converged (and separation of signals is established), the parameters $a_{i j}$ are then varied in order to observe the quality of performance. Experiments for different starting values of the mixing matrix $A$ were also performed. The percentage of robustness to each parameter is then recorded. Based on the experiments, the network is robust to parameter variations of about $15 \%$ (on average).

\subsubsection{Limitations}

As the level of mixing is varied, it is discovered that the network fails to separate the signals when the mixing reaches a certain level. Based on experiments, the normalized coefficients of the mixing 
matrix A should range between 0.0 and 0.8 for the first four scenarios and between 0.0 and 0.3 for the fifth scenario in order to achieve successful signal separation. Observe that this limitation is inherent in the algorithms themselves as has been evidence by computer simulations.

\subsection{The Dynamic Modeling Case}

We now consider that the input to the network is a superposition and a delayed (filtered) version of the unknown sources. This is a more realistic, real-world, mixing scenario. The chip failure to perform separation is not due to chip nonidealities but rather due to the algorithm itself. The same limitations had been observed in computer simulations.

\subsubsection{Experimental Setup}

The circuit diagrams shown in Figure 6 (a) is used to obtain a filtered and mixed version of the original signals. Specifically each measured signal $e_{i}$ is given by

$$
e_{i}=\sum_{j=1}^{n} F_{i j}\left(s_{j}\right)
$$

where $F_{i j}($.$) is a low pass filter with gain g_{i j}$ and cutoff frequency $\omega_{i j}$

$$
F_{i j}\left(V_{i n}\right)=\frac{g_{i j}}{\frac{s}{\omega_{c i j}}+1} V_{i n}
$$

Observe that $V_{i n}$ represents the Laplace transform of an input signal, say, $s_{j}(t)$. The mixing and filtering constants are given in terms of the circuit elements as:

$$
g_{i j}=\frac{R_{0}}{R_{i j}} \quad \omega_{c i j}=\frac{1}{R_{0} C_{F i j}}
$$

For our experiments $R_{0}=10 k \Omega$ and $0.1 n F<C_{F i j}<0.1 m F$. Thus the cutoff frequency range is $1<\omega_{c i j}<10^{6}$.

\subsubsection{Experimental Results}

The goal now is to study whether the modified algorithm can still update the parameters and achieve convergence. The parameters $d_{i j}$ must adaptively counter the effect of the mixing and filtering, and thus recover the original signals. The five previous scenarios are repeated in studying this problem. Exhaustive experimentation and testing led to the conclusion that separation of the signals occurs in the first four scenarios when $g_{i j}<0.4$ and also in the fifth scenario when $g_{i j}<0.15$. Figures 11 12 depict some sample test results. The normalized range of parameter variations as a robustness 
measure is now more restrictive than in the static medium case. However, the normalized ranges specify the parameter intervals where separation of signals can be performed reliably.

\subsubsection{Limitations}

It is noted that the interval range over which the separation of signals in the dynamic medium case had shrunk considerably in comparison to the linear static medium case. Nonetheless, the chip was able to achieve signal separation in this dynamic medium as well. The mixing parameter

$g_{i j}<0.4$ for the cutoff frequency range $1<\omega_{c i j}<10^{6}$ may be acceptable for a wide range of applications which seek to enhance or purify the quality of signals. However it may be limited in other applications where normalized mixing exceeds 0.4 .

\section{Conclusion}

We have designed, implemented and tested a CMOS chip for a modified HJ algorithm and explored its validity range. The modification produces robust performance to parameter variations and to dynamic media effects. Limits were also experimentally quantified. The fact that realistic sensors have their own inherent dynamics underlines the need for such considerations. We showed that the modified algorithm has a potential in solving the problem of SIS in more realistic real-world environments.

\section{References}

[1] J. Herault and C. Jutten. Blind separation of sources, part 1: An adaptive algorithm based on neuromimetic architecture. Signal Processing, 24:1-10, July 1991.

[2] J. Herault and C. Jutten. Blind separation of sources, part 2: Problem statement. Signal Processing, 24:11-20, July 1991.

[3] J. C. Platt and F. Faggin. Networks for the separation sources that are superimposed and delayed. Advances in Neural Information Processing systems, 1:730-737, 1992.

[4] B. Widrow et al. Adaptive noise cancellation: principles and applications. IEEE Proceedings, 63:1691-1717, April 1975. 
[5] F. M. A. Salam. An adaptive network for blind separation of independent signals. International Symposium on Circuits and Systems, 1:431-434, May 1993.

[6] K. S. Narendra and K. Parthasaraphy. Identification and control of dynamical systems using neural networks. IEEE Transactions on Neural Networks, 1:4-27, March 1991.

[7] E. Vittoz and X. Arreguit. Cmos integration of herault-jutten cells for separation of sources. Proceedings Workshop on Analog VLSI and Neural Systems, May 1989.

[8] M. H. Cohen and G. Andreou. Current-mode subthreshold mos implementation of heraultjutten autoadaptive network. IEEE Journal of Solid-State Circuits, 27(5):714-727, May 1992.

[9] C. Jutten. Calcul neuromimétique et traitement du signal, analyse en composantes indépendantes. Thèse de doctorat d'etat, Université Scientifique et Médicale-Institut Nationale Polytechnique, Grenoble, France, 1987.

[10] C. Mead. Analog VLSI and Neural Systems. Prentice Hall, New York, 1989.

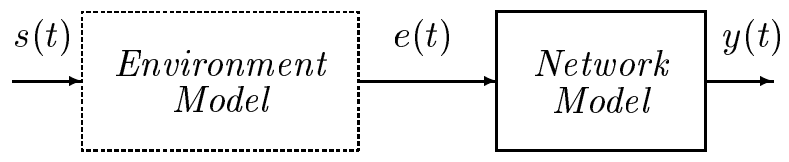

Figure 1: Block diagram 

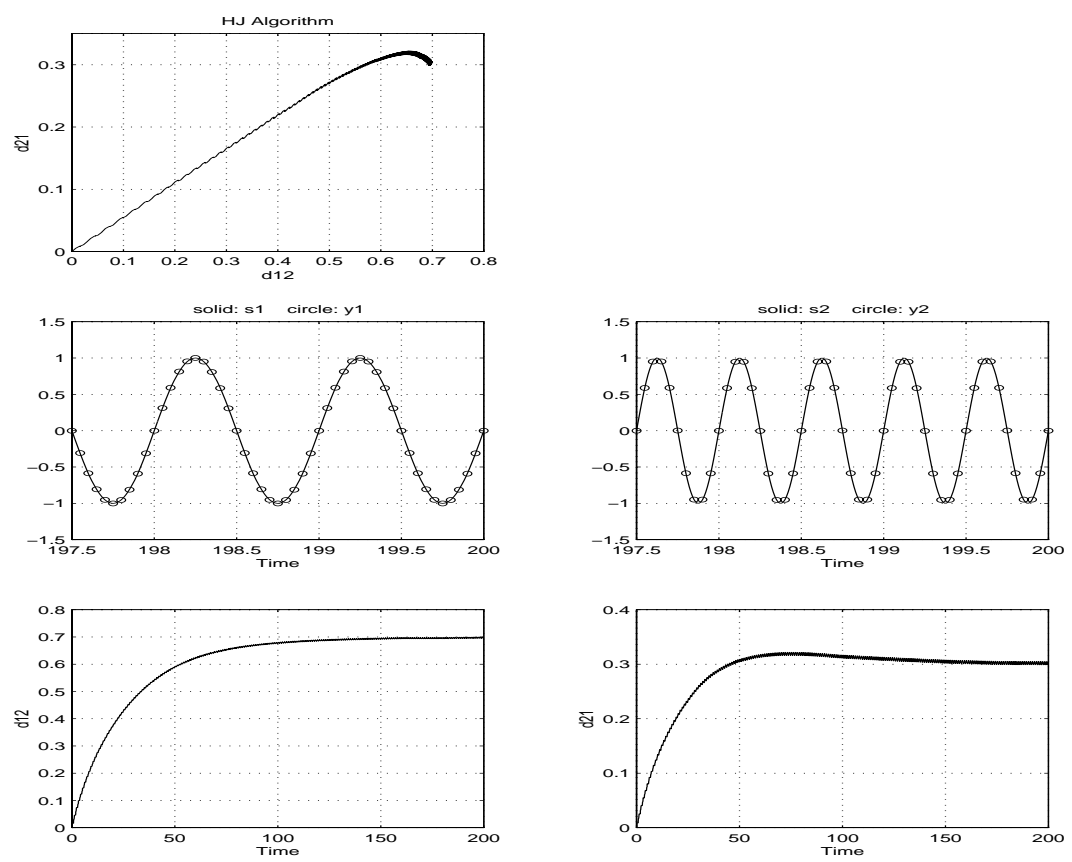

(a) HJ algorithm with $\eta=0.05$
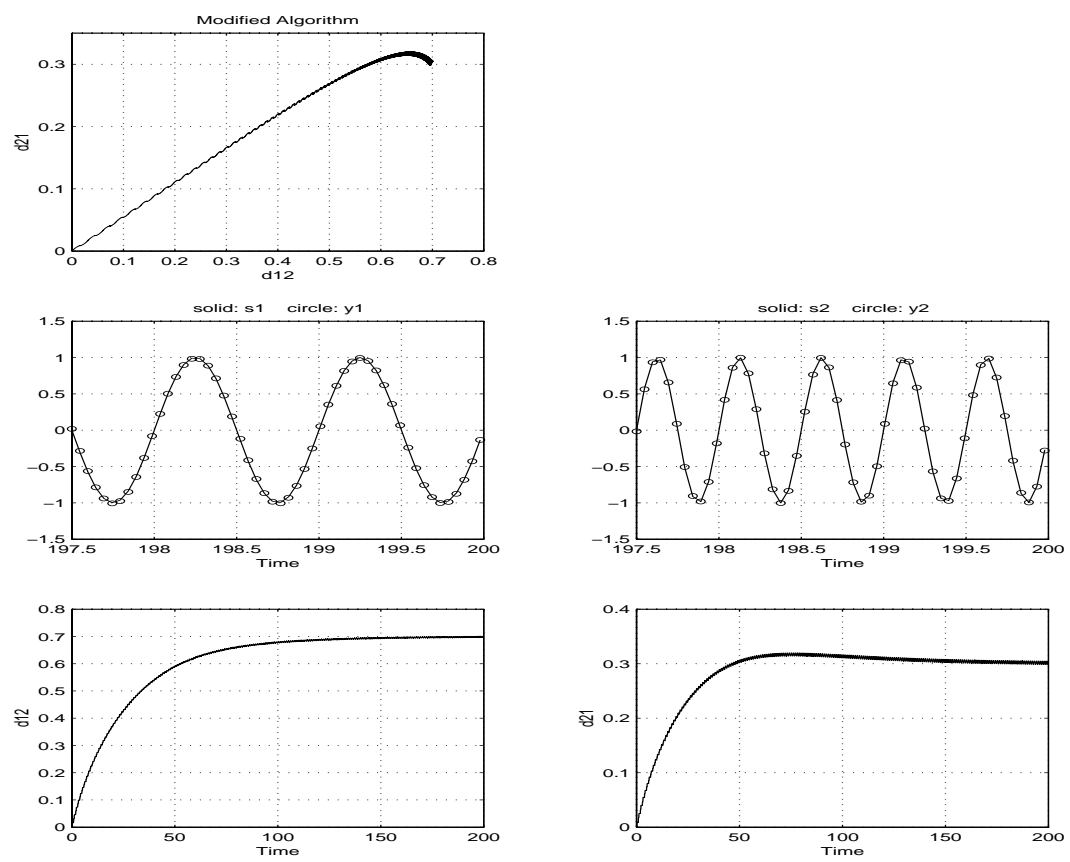

(b) Modified HJ Algorithm with $\eta=0.05$ and $\tau=0.001$

Figure 2: Computer Simulations of Algorithms 

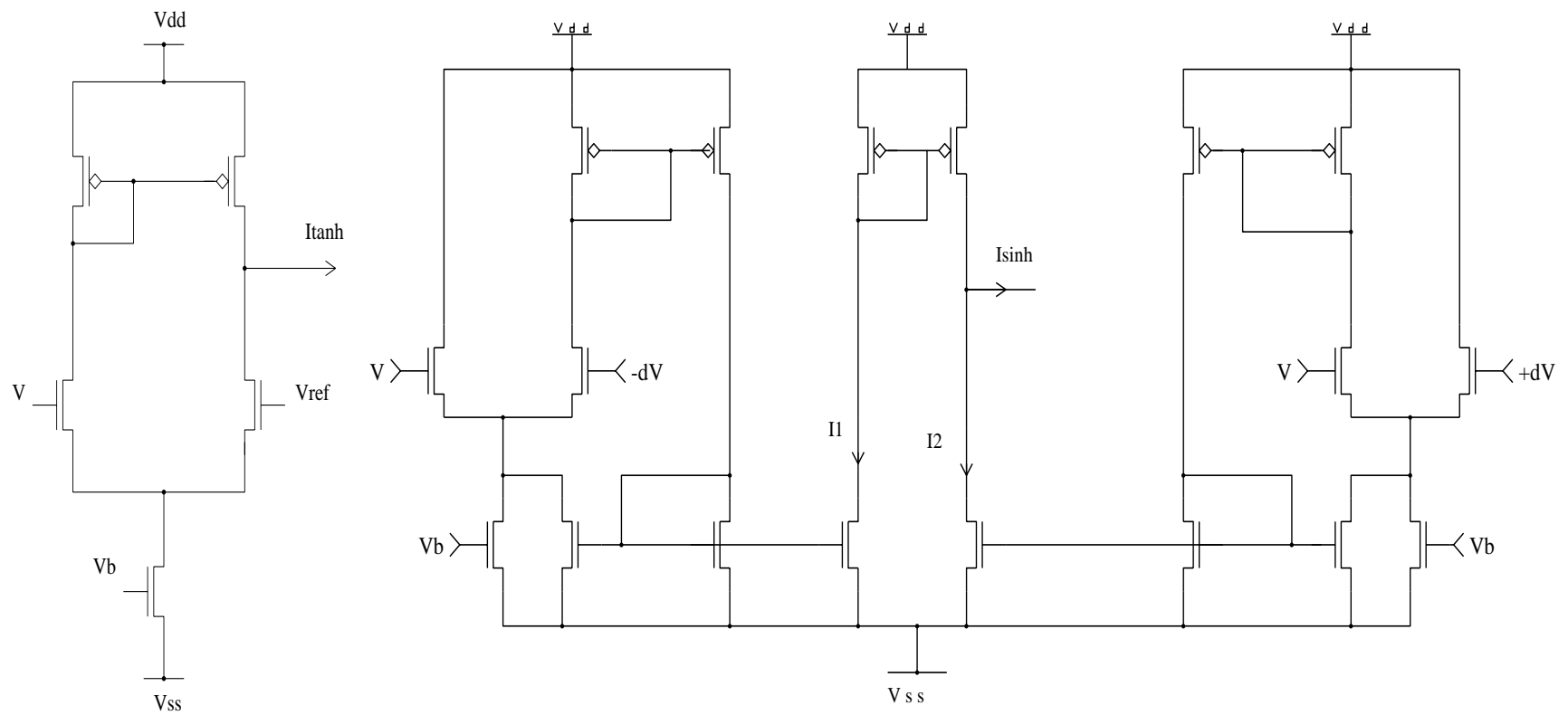

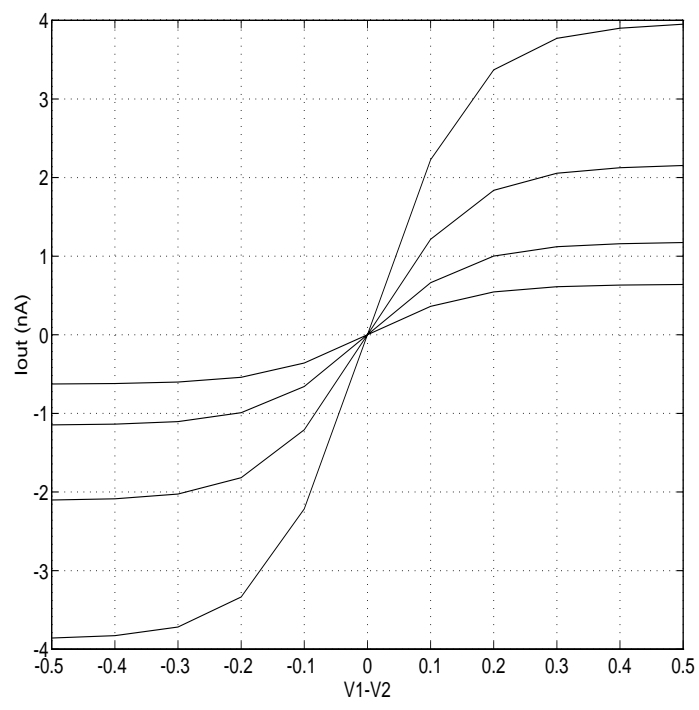

Hyperbolic tangent

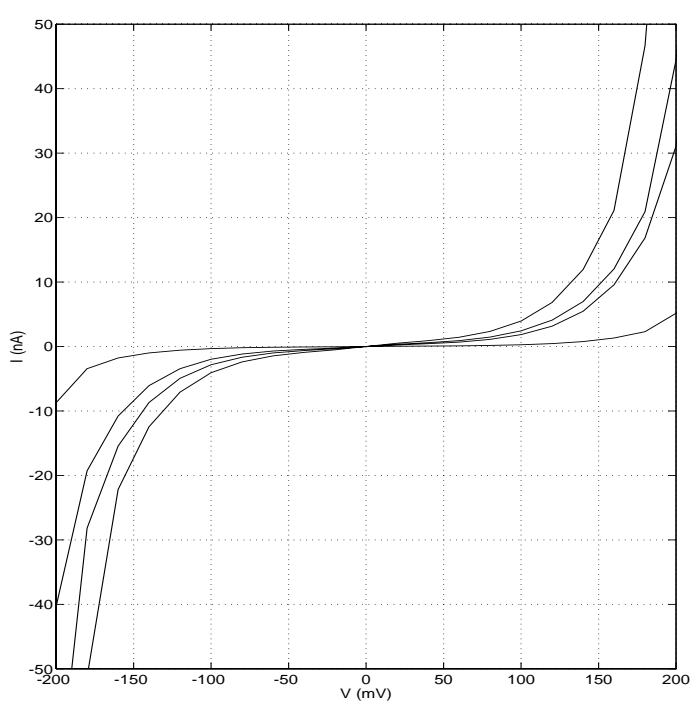

Hyperbolic sine

Figure 3: Basic Analog Components 


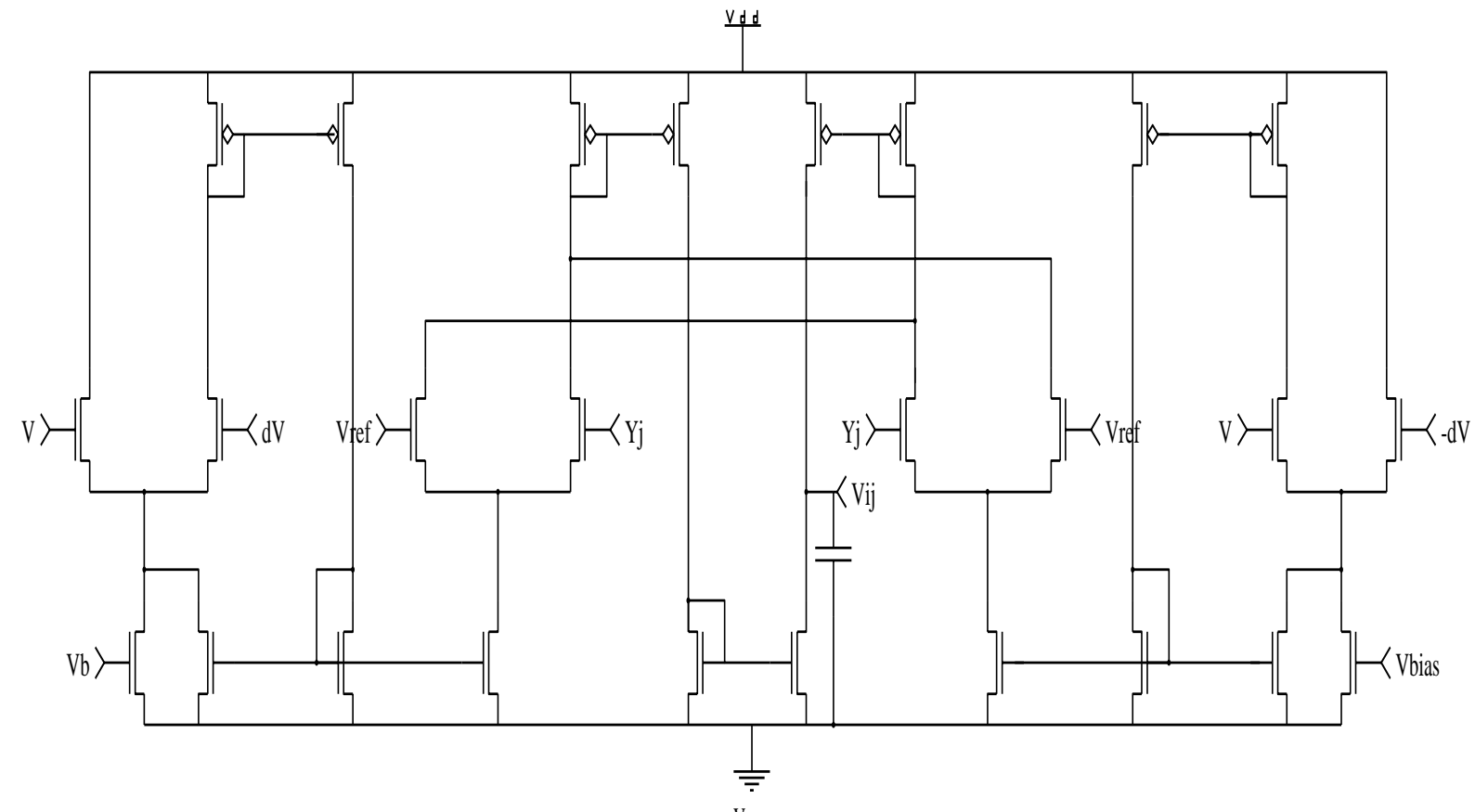

(a) Circuit Implementation of (3)

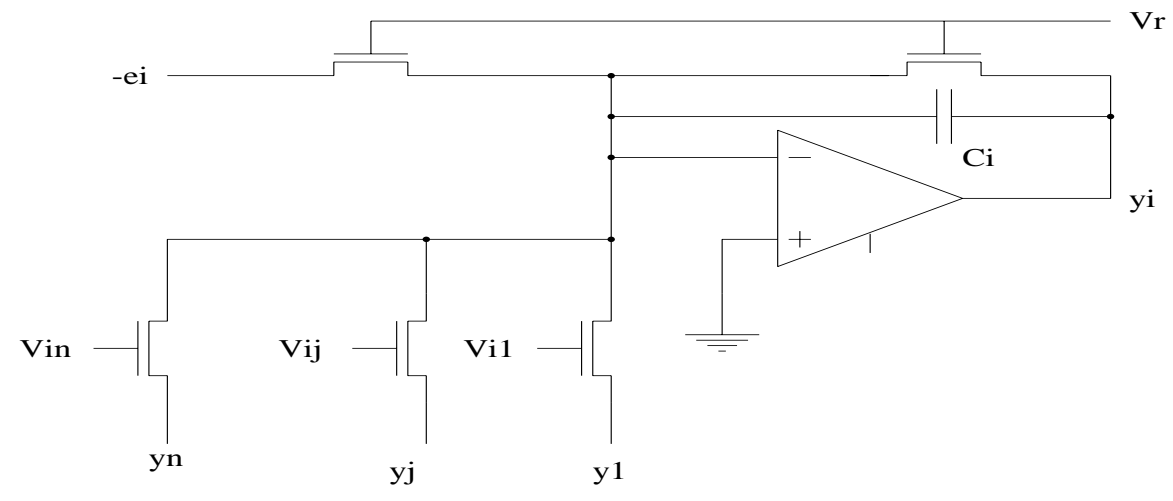

(b) Circuit Implementation of (4)

Figure 4: Circuit Implementation 
Modified HJ

Date/Time run: 11/07/94 03:52:19

Temperature: 27.0
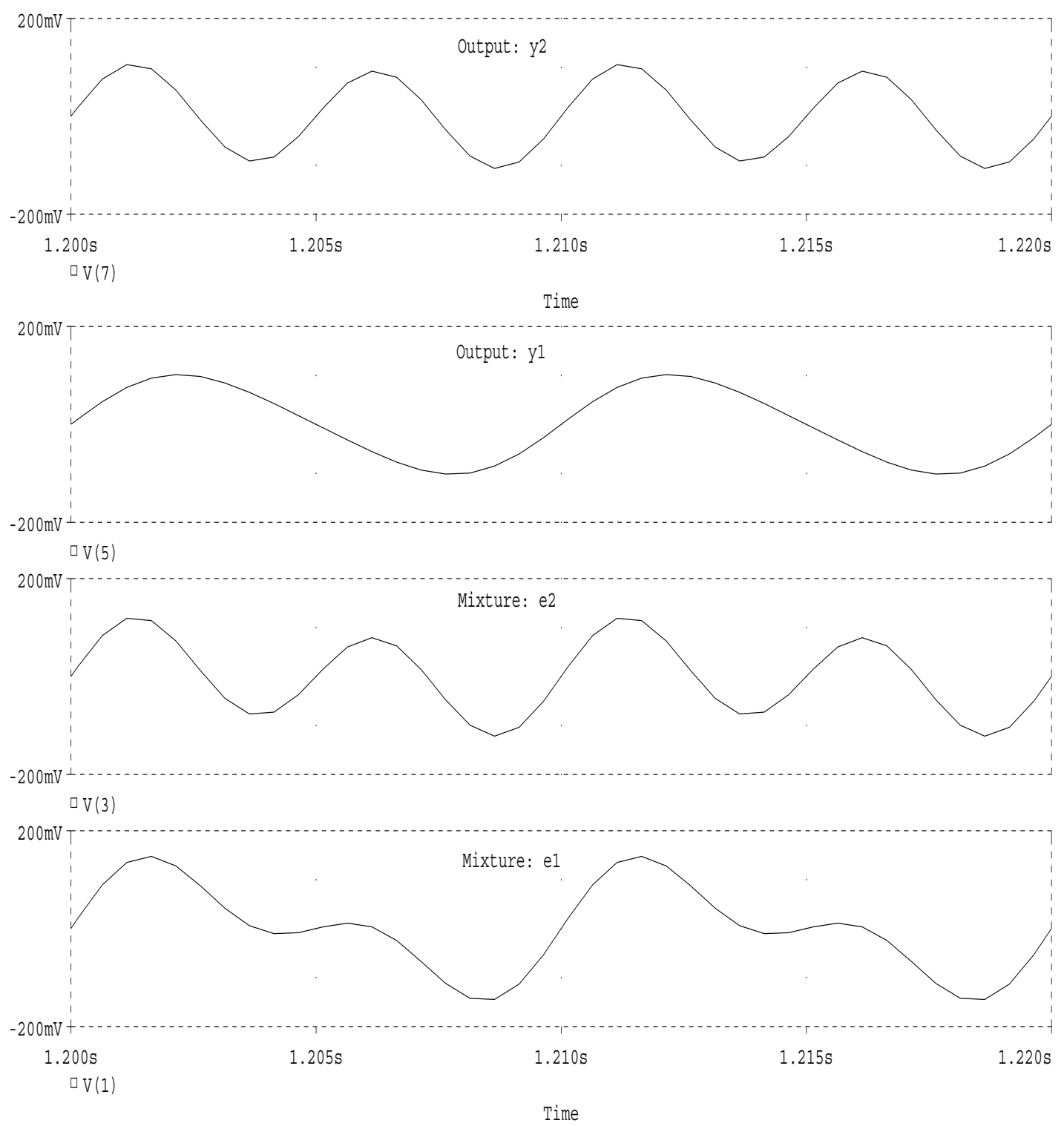

Figure 5: PSPICE Simulations 


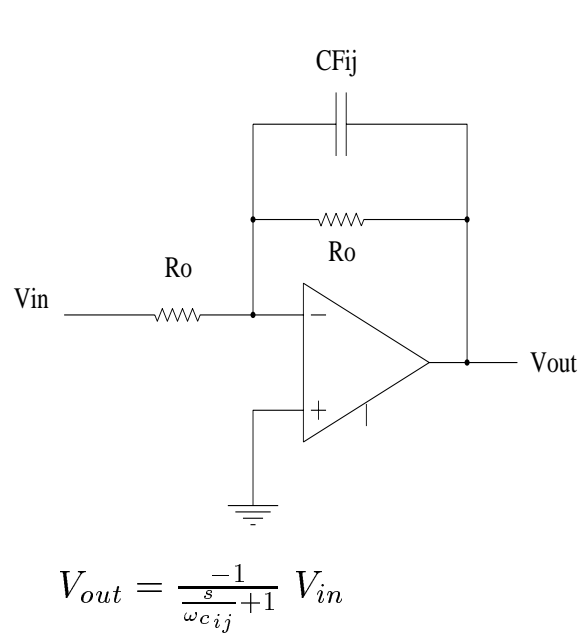

(a) Low pass filter

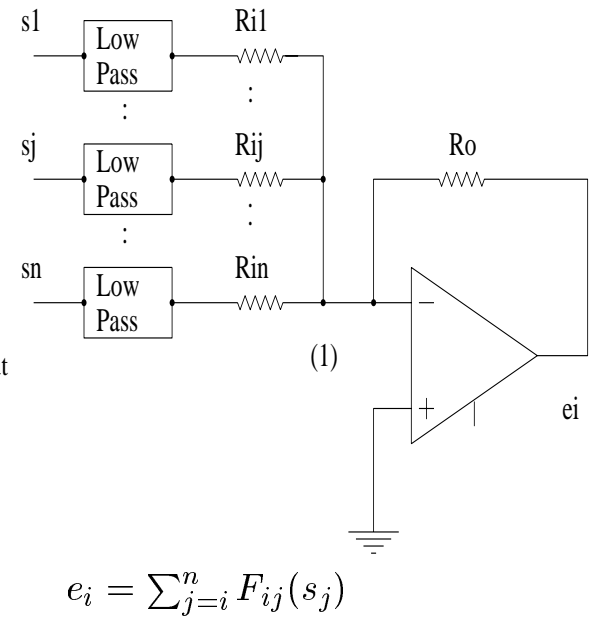

(b) Mixing/filtering circuit

Figure 6: Mixing Circuit 

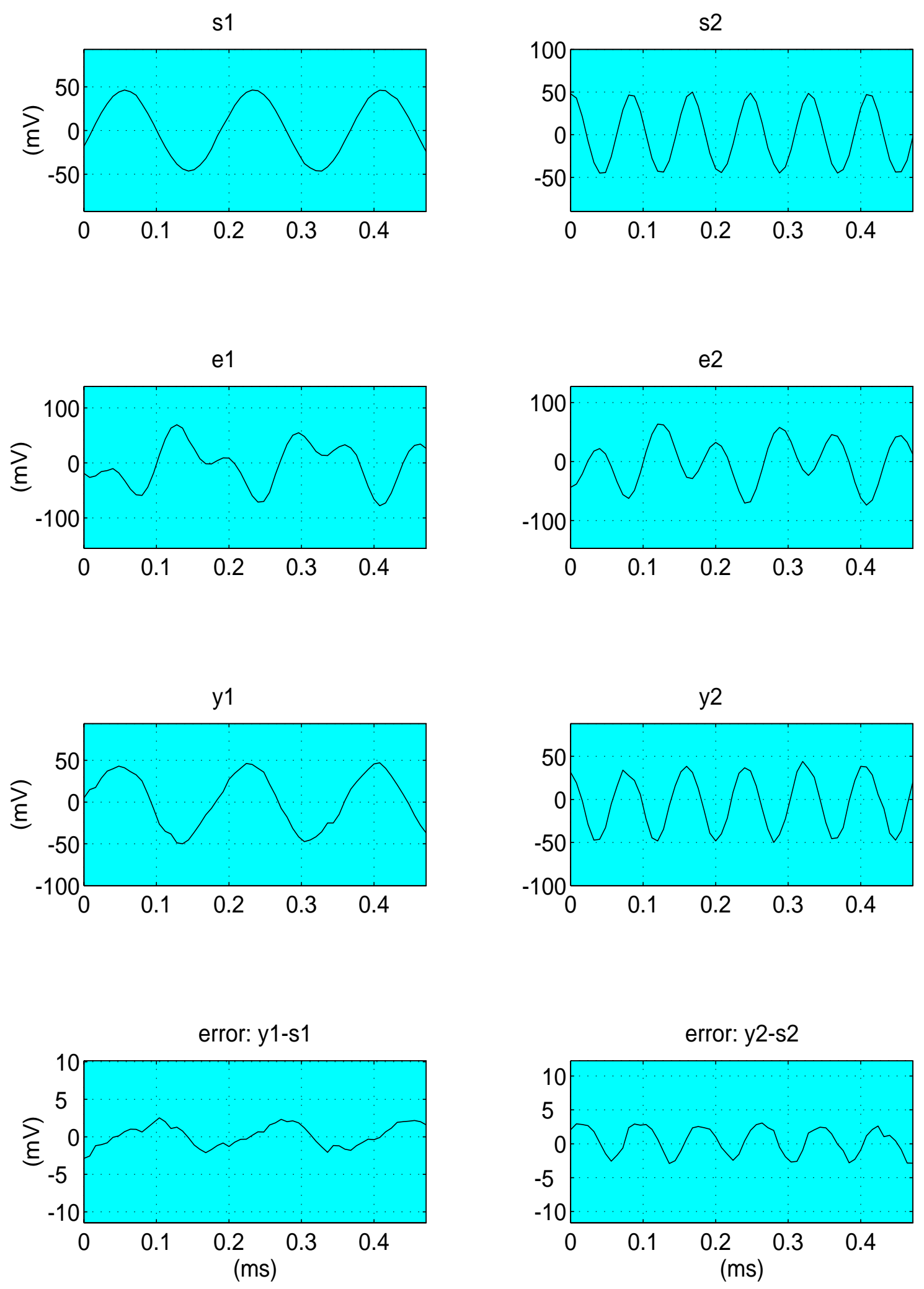

Figure 7: Static Mixing of two sine functions 

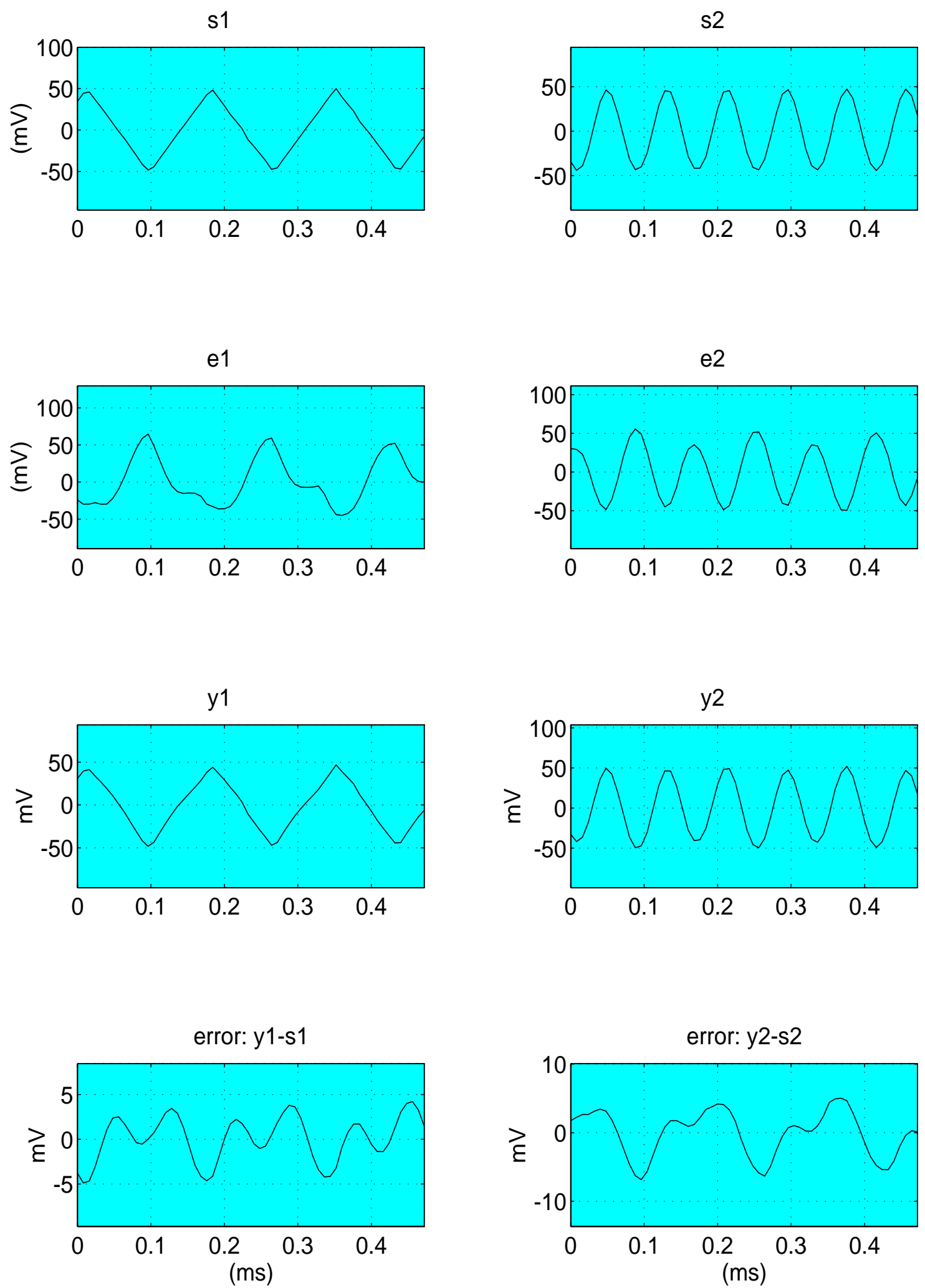

Figure 8: Static Mixing sine and triangular functions 

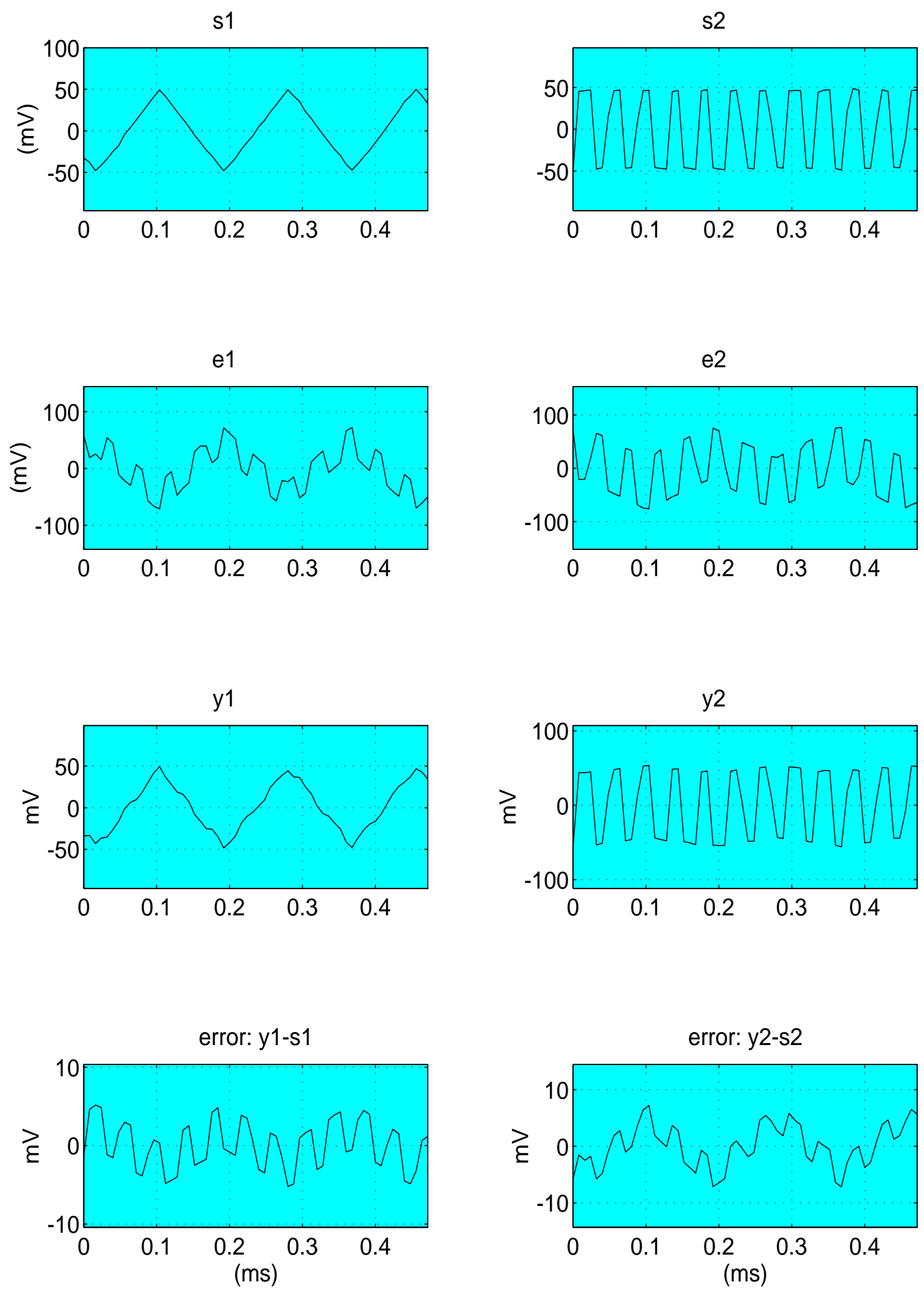

Figure 9: Static Mixing of triangular and square functions 

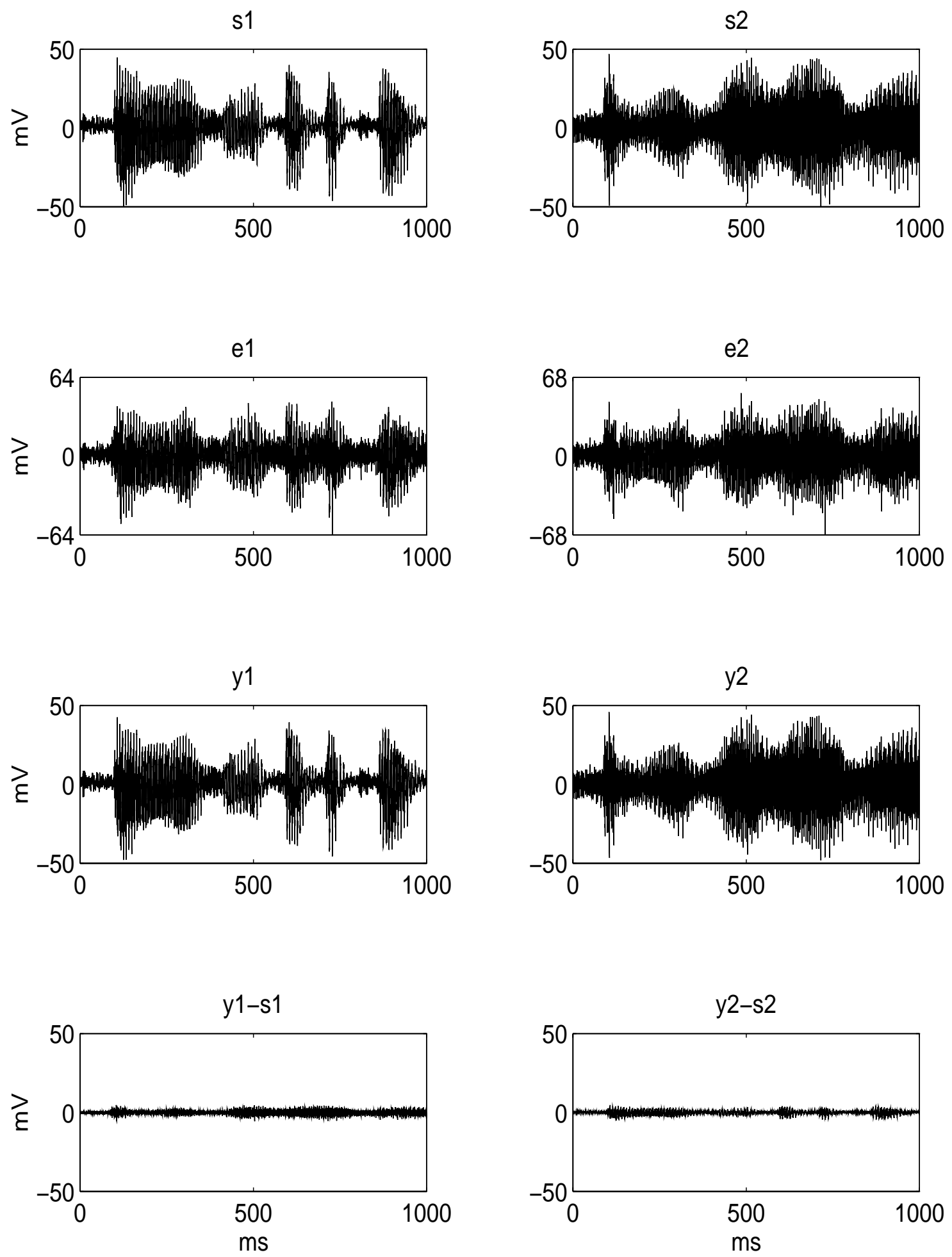

(a) Japanese and English segments

Figure 10: Static Mixing 

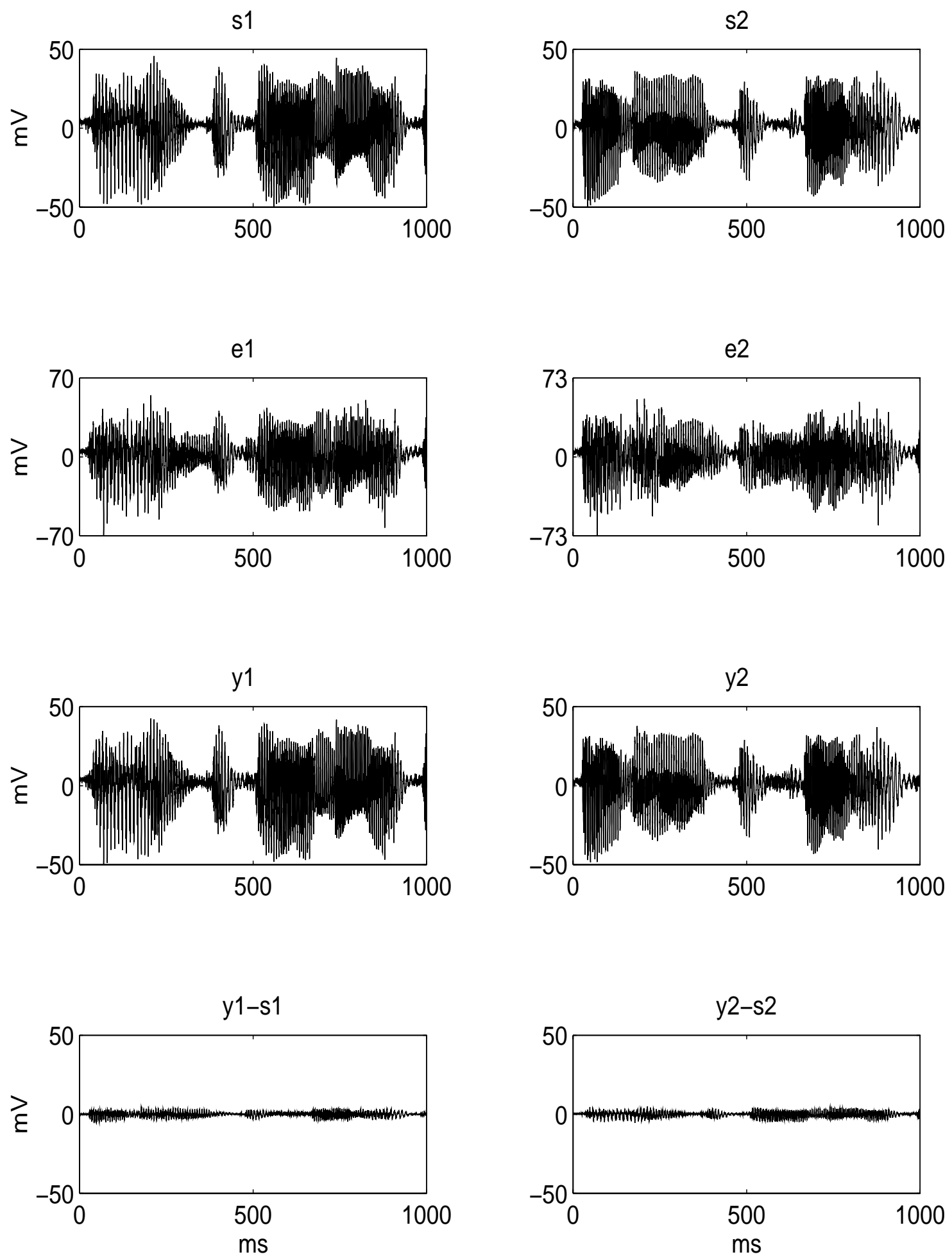

(b) Both English segments

Figure 10: Static Mixing 

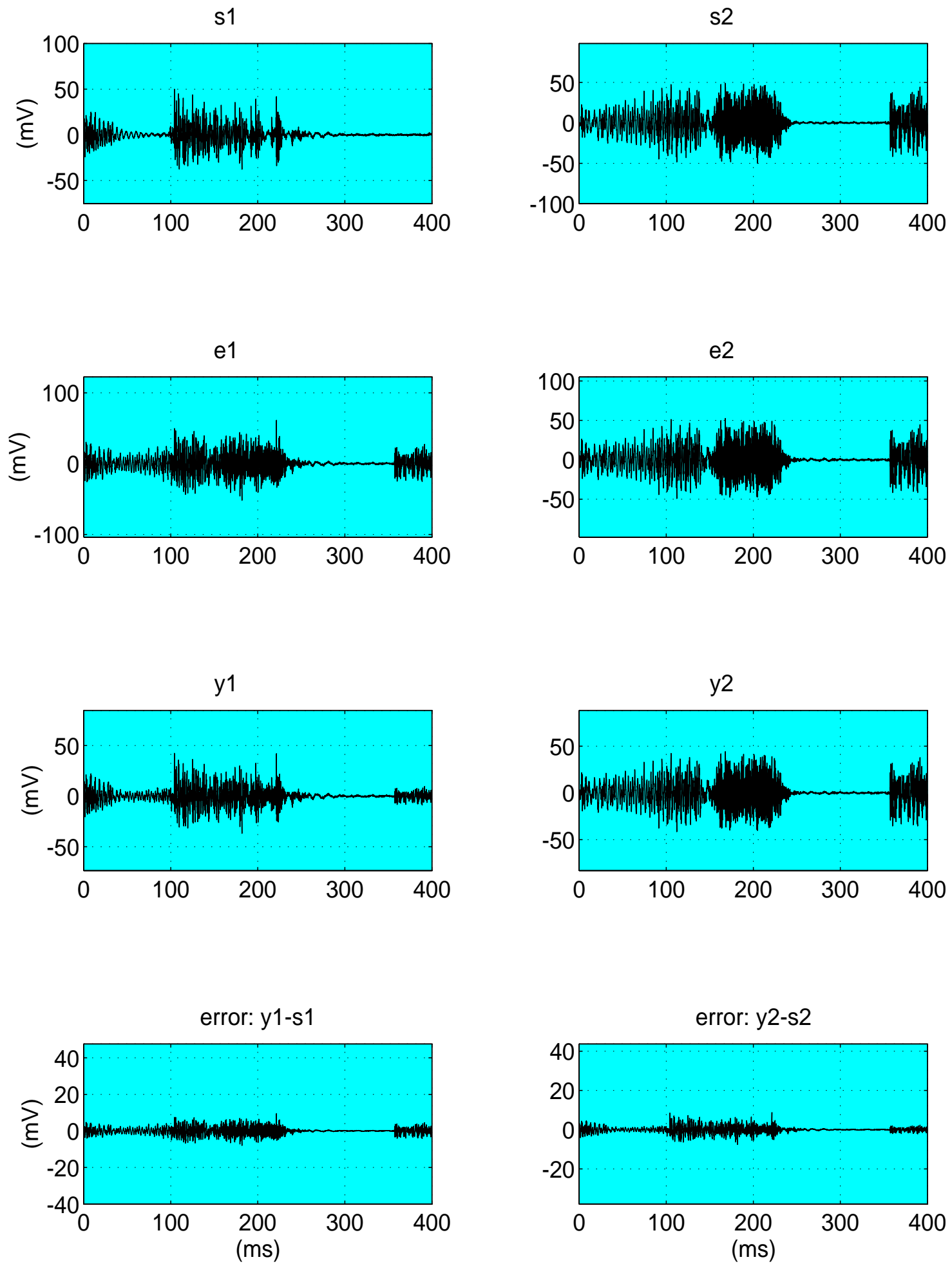

(c) Arabic and English segments

Figure 10: Static Mixing 

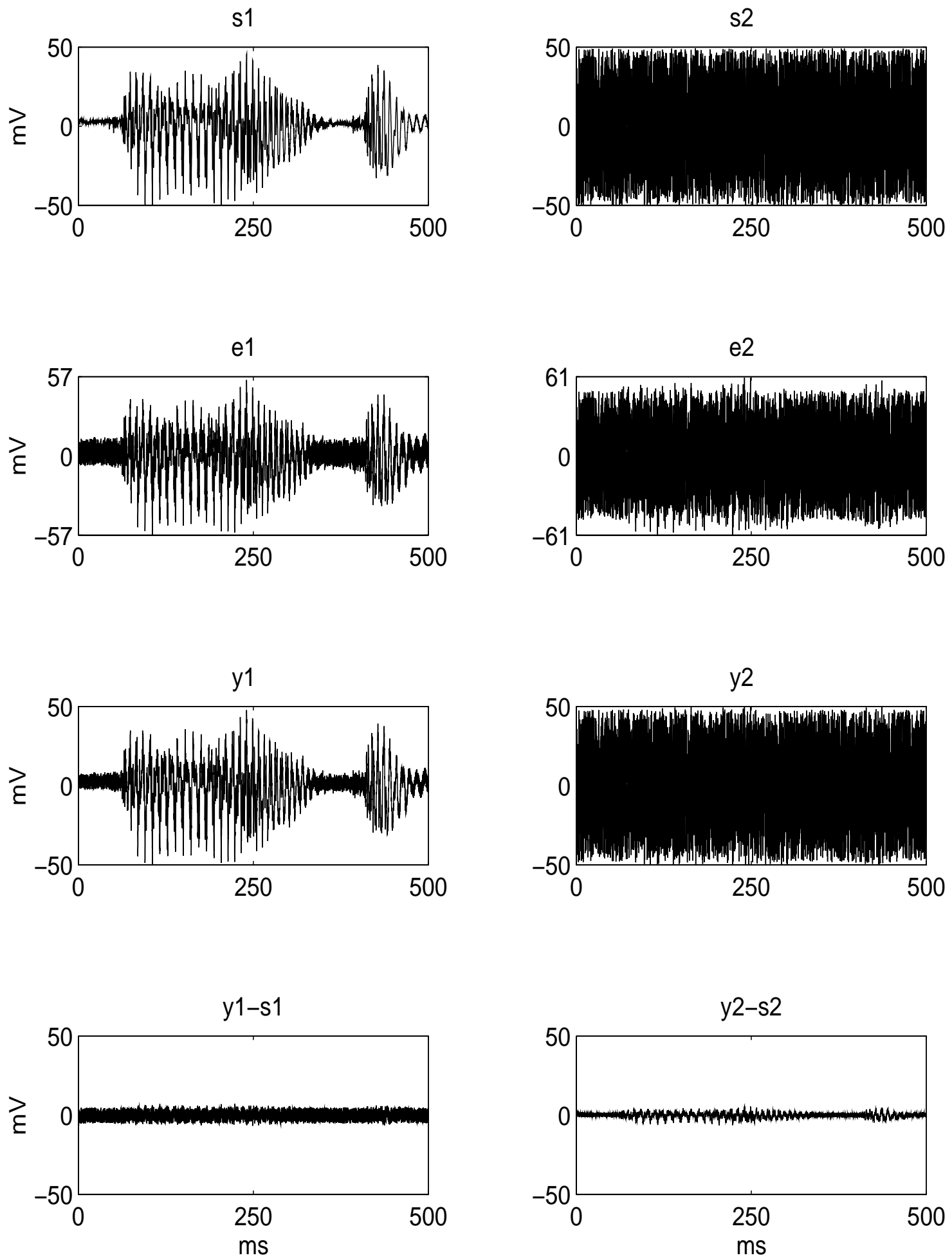

(d) White signal and English segment

Figure 10: Static Mixing 
s1
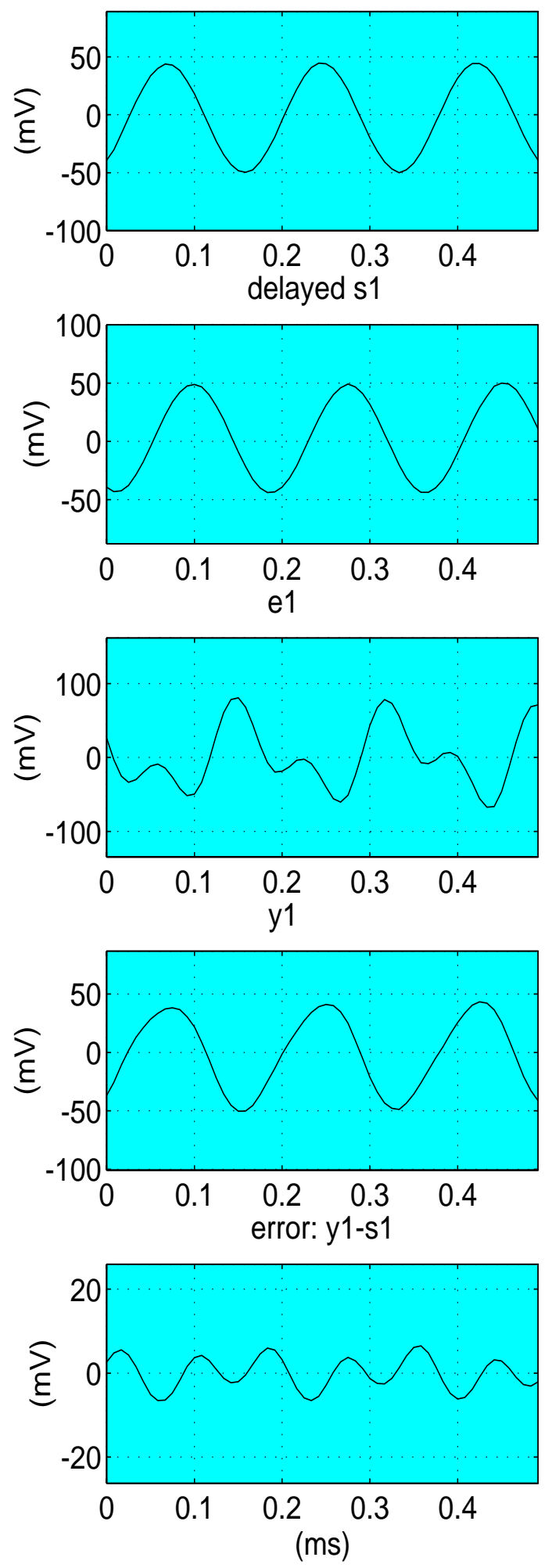

s2
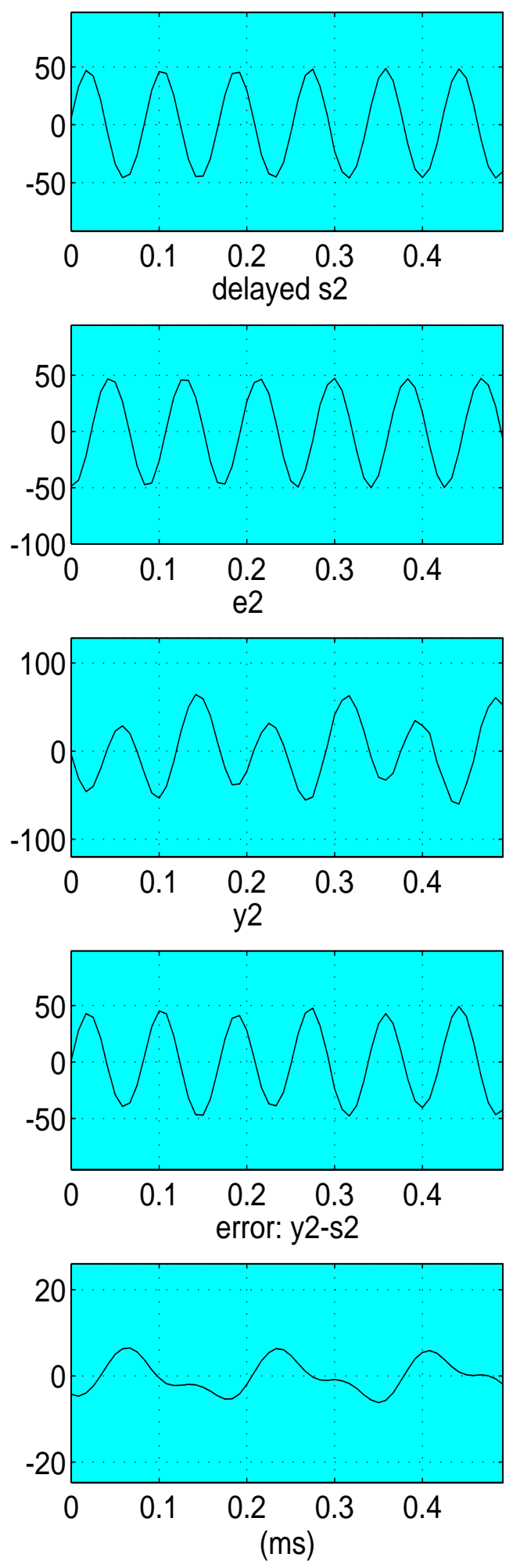

Figure 11: Dynamic Mixing of two sine functions 

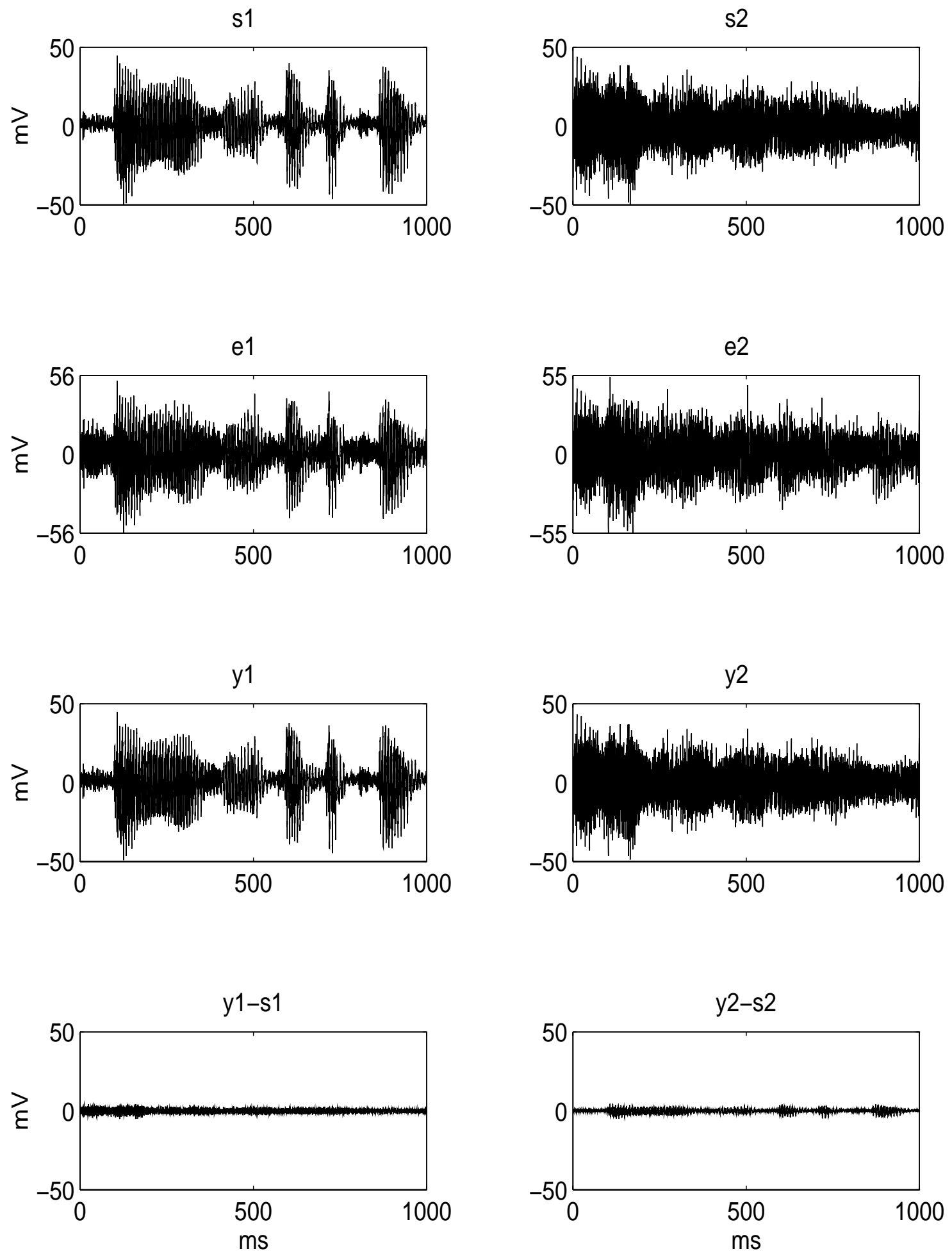

(a) Japanese and English segments

Figure 12: Dynamic Mixing 

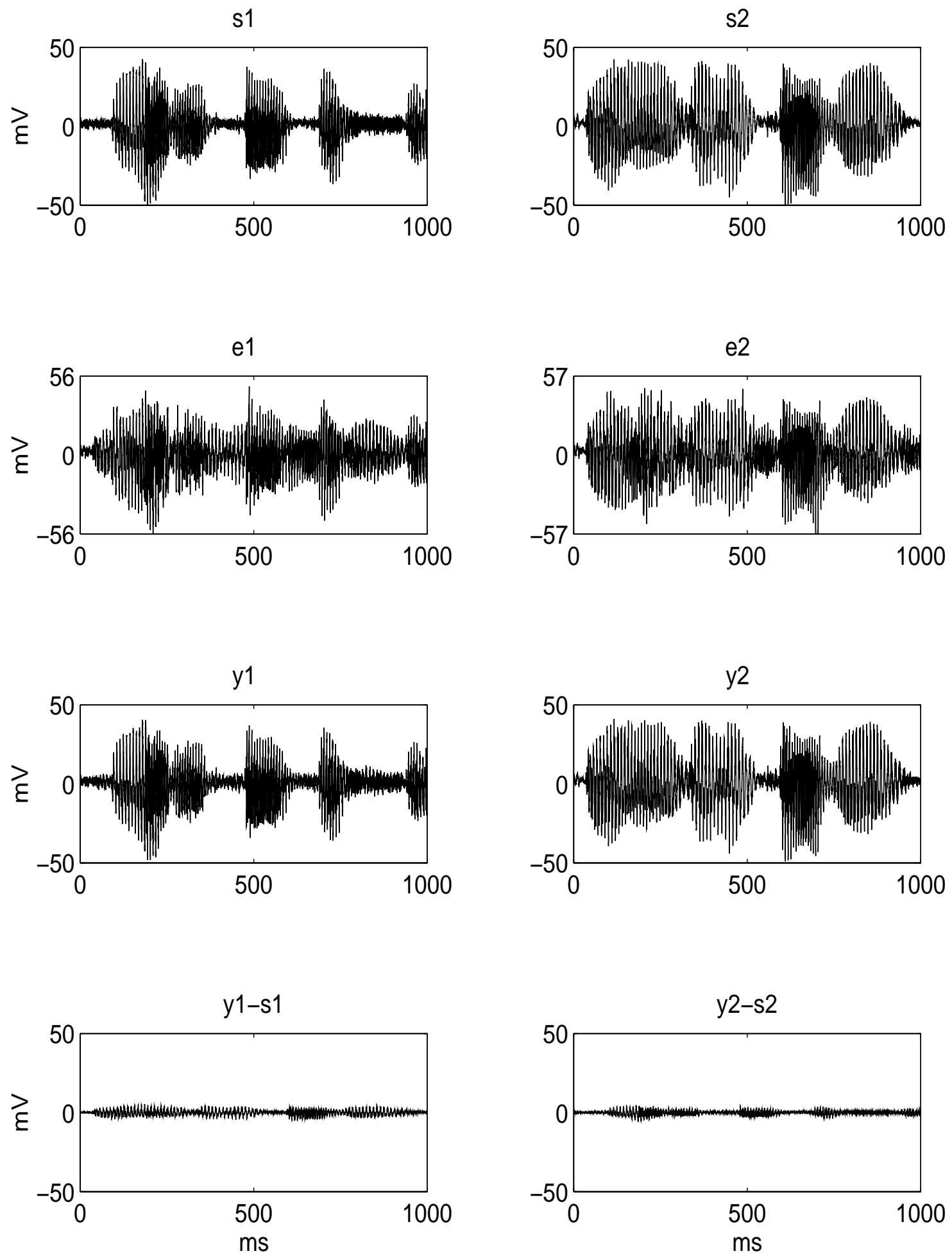

(b) Both English segments

Figure 12: Dynamic Mixing 

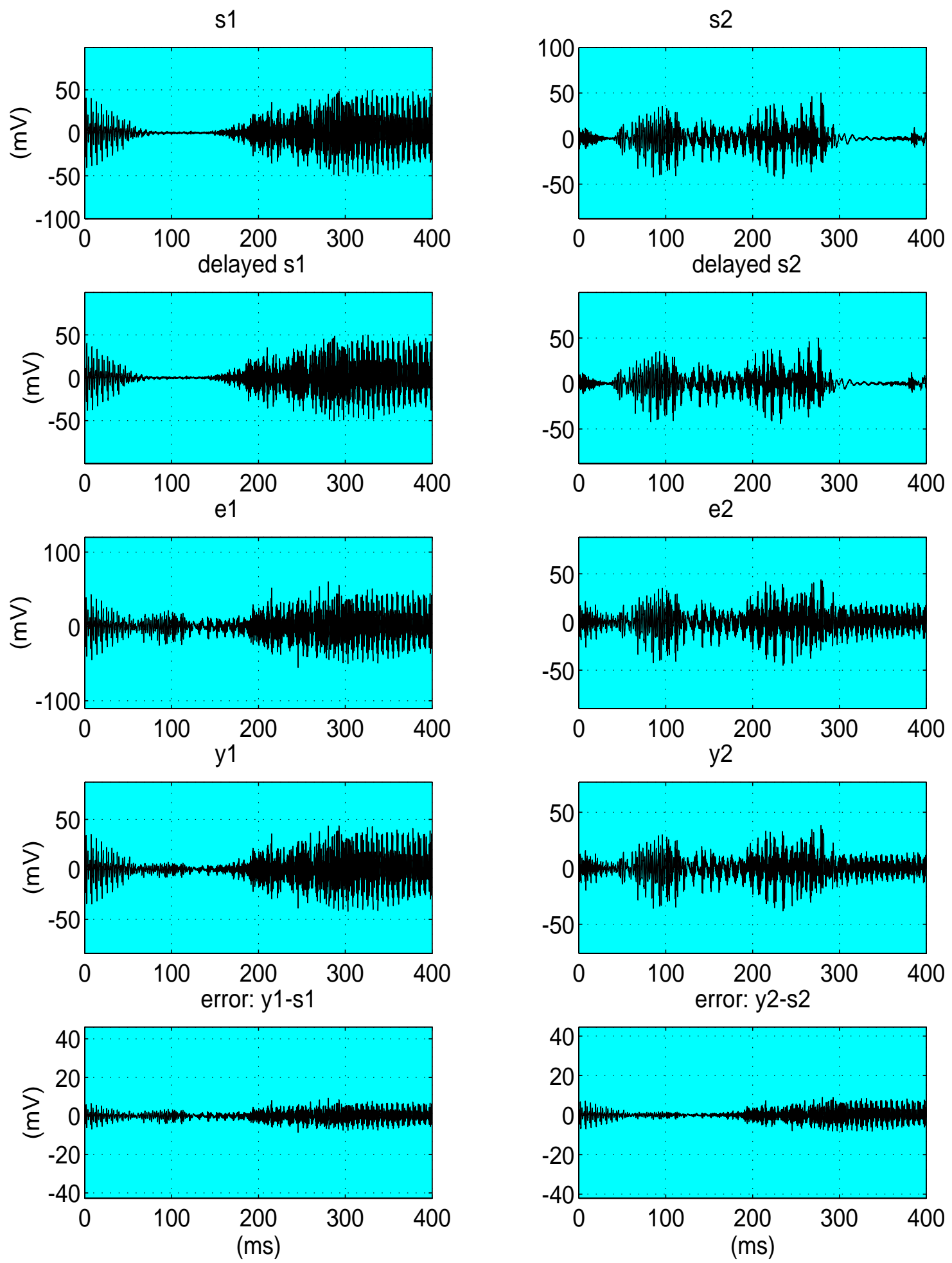

(c) Arabic and English segments

Figure 12: Dynamic Mixing 